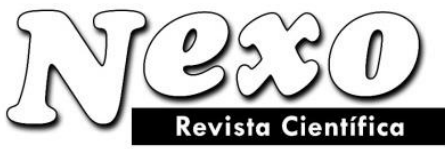

ISSN-E 1995-9516

Universidad Nacional de Ingeniería COPYRIGHT (C) (UNI). TODOS LOS DERECHOS RESERVADOS http://revistas.uni.edu.ni/index.php/Nexo https://doi.org/10.5377/nexo.v32i01.7989

\title{
DISEÑO DE UNA PROPUESTA DE UNA PLANTA INDUSTRIAL PARA EL APROVECHAMIENTO DE RESIDUOS SÓLIDOS DEL PROCESO DE CURTIDO
}

\section{DESIGN OF A PROPOSAL FOR AN INDUSTRIAL PLANT FOR THE USE OF SOLID RESIDUES OF THE TANNING PROCESS}

\author{
K.E. Medina Ninacondor*, J.A. Aguilar Franco, J. Villegas Alvarez \\ ${ }^{1}$ Universidad Católica San Pablo. Instituto de Energía y Medio Ambiente. Arequipa, Perú. \\ *katherine.medina@ucsp.edu.pe
}

(recibido/received: 12-Septiembre-2018; aceptado/accepted: 29-Octubre-2018)

\begin{abstract}
RESUMEN
En este artículo se presentará el diseño e implementación de una planta en una empresa industrial que permita el reaprovechamiento de los residuos sólidos (viruta de cuero) del proceso de curtido durante el proceso de elaboración de cueros para su utilización en un proceso complementario. Los problemas que se presentan al no contar con este tipo de procesos es incurrir en costos extras para tercerizar servicios para la disposición de estos desechos contribuyendo al aumento de la contaminación en la ciudad. Para implantar esta mejora será necesario realizar un análisis preliminar para poder elaborar la propuesta de diseño de planta más óptima que se adecúe a este proceso. La línea productiva está constituida por cuatro Unidades, la primera Unidad de Acondicionamiento de materia prima; la segunda Unidad de Mezclado y Moldeado; la tercera Unidad de Prensado y Secado, y finalmente la Unidad de Acabado.
\end{abstract}

Palabras claves: Distribución de planta, Residuos Sólidos, Viruta de cuero, Cuero Regenerado.

\begin{abstract}
This article will present the design and implementation of a plant in an industrial company that allows the reuse of solid waste from the tanning process during the process of making hides for use in a complementary process. The problems that arise when not having this type of process is incurring in extra costs to outsource services for the disposal of these wastes, contributing to the increase of pollution in the city. To implement this improvement, it will be necessary to carry out a preliminary analysis to be able to elaborate the most optimal plant design proposal that fits this process. The production line is made up of four Units, the first Raw Material Conditioning Unit; the second Mixing and Molding Unit; the third Pressing and Drying Unit, and finally the Finishing Unit.
\end{abstract}

Keywords: Plant distribution, Solid Waste, Leather chip, Regenerated Leather. 


\section{INTRODUCCIÓN}

La presente investigación abarca el diseño e implementación de una planta para el reaprovechamiento de residuos sólidos (viruta de cuero) generados en el proceso de curtido de una curtiembre, para la obtención de un producto alternativo (cuero regenerado). Las empresas de curtiembres como todo proceso industrial generan residuos sólidos y líquidos en sus diferentes etapas, los cuales requieren horas-hombre para su traslado y disposición, uso de áreas temporales para el almacenamiento y costos adicionales en la

contratación de EPS (Empresas Prestadoras de Servicios de Residuos Sólidos) para su disposición final hacia el relleno sanitario (Dirección General de Salud Ambiental, 2004).

Los residuos sólidos representan alrededor del $40 \%$ de la materia prima y están conformados por carnazas, gamuzón, pelos, restos de carne y viruta de cuero, éstos últimos son los más relevantes ya que la empresa genera $3200 \mathrm{~kg}$ de viruta para una producción mensual de 804 mantas de cuero, representando un poco más del 50\% de los residuos en la curtiembre. Hoy por hoy estos residuos una vez generados son depositados en saquillos y almacenados temporalmente tras el proceso de rebajado, en lo posterior (fines de semana) con ayuda del montacargas son trasladados al almacén general de viruta sin obstruir ningún tipo de vía de acceso ni generar contaminación durante su almacenamiento, finalmente la EPS contratada se encarga de pesarlos y llevarlos hacia el relleno sanitario con una periodicidad trimestral.

En los últimos meses la empresa en su búsqueda de alternativas y soluciones para la disposición o eliminación de éstos residuos para cumplir con la normativa vigente y la ley general de residuos sólidos ha encontrado investigaciones y artículos donde se evidencia la gran potencialidad para tratarlos, reutilizarlos y/o reaprovecharlos para fabricar productos secundarios, este es el caso de las virutas de cuero que son residuos considerados de menor valor y desechos, sin embargo se ha constatado que pueden ser reaprovechados, reutilizados o tratados para obtener un producto alternativo.

En caso las empresas de curtiembres opten por el reaprovechamiento del residuo sólido (viruta de cuero) eso demanda el desarrollo de un nuevo proceso, el cual va a consistir en el diseño e implementación de una nueva línea productiva considerando áreas, distribuciones, acondicionamientos de ambientes, recursos, maquinarias, equipos, herramientas, mano de obra, entre otros factores que permitirán realizar los estudios de factibilidad y viabilidad de la línea/planta productiva.

Para el diseño e implementación de la planta se ha realizado un estudio previo empleando técnicas de ingeniería, análisis, cálculos, diagramas, entre otros, que han permitido el dimensionamiento del diseño de la planta (Contreras, 2004). Además, se ha considerado lineamientos establecidos por el Reglamento Nacional de Edificaciones que involucra edificaciones, arquitectura, instalaciones eléctricas, mecánicas y sanitarias, estructuras, entre otros. Se hizo hincapié principalmente en dos normas: la Norma A.010 que cita las Condiciones Generales de Diseño; y, la Norma A. 060 que hace referencia a la Industria, ambas detallan aspectos generales para la edificación de una planta industrial, los cuales han sido aplicados también para la industria de curtido.

\section{METODOLOGÍA}

La metodología experimental propuesta para el diseño y modelado de una planta industrial para la implementación de una línea productiva para el aprovechamiento de residuos sólidos (viruta de cuero) del proceso de curtido, se ha diseñado con la finalidad de cumplir los objetivos planteados a través del estudio técnico.

Se inició con la elección de la localización la cual ya se encuentra identificada ya que la línea productiva se situará dentro de las instalaciones de la planta actual, por consiguiente, se procedió a la determinación de la ubicación más adecuada para albergar la implementación de la línea, para el análisis respectivo, se 
identificó dos alternativas potenciales que podían ser utilizadas. La primera de ellas, ubicada en el primer nivel de la planta cercana al área de acabados y; la segunda, ubicada en el segundo nivel de la planta. El análisis para la identificación de la ubicación más óptima contempló el uso del método de factores ponderados, para este estudio fueron analizados 5 factores (proximidad a la materia prima, abastecimiento de servicios complementarios, disponibilidad de área superficial de ambiente de trabajo, distancia del proceso y, costos administrativos y operativos) que guardan una relación directa con la instalación e implementación de la línea productiva, teniendo como resultado que la alternativa ubicada en el primer nivel cuenta con mayor proximidad a los servicios complementarios, menor proximidad a la materia prima incurriendo en mayor tiempo de abastecimiento, dispone de mayor área superficial, cuenta con menor distancia de recorrido durante todo el proceso e incurre en menores costos administrativos y operativos.

Seguidamente se determinó el tamaño de planta tomando ciertas consideraciones previas que sirvieron para determinar que el tamaño será de $6600 \mathrm{~kg}$ de residuos sólidos (viruta de cuero) por mes que representa aproximadamente una producción mensual de 812 planchas de cuero considerando como criterio un incremento en la producción actual de un $37 \%$ los próximos 5 años. El cálculo se realizará a continuación:

$$
\text { Cant.residuos sólidos generados }=4800 \mathrm{~kg}
$$

Considerando el crecimiento de la producción en un 37\%:

$$
\text { Cant.residuos sólidos por generar }=4800 \times 1.37=6576 \mathrm{~kg} \approx 6600 \mathrm{~kg}
$$

Asimismo, para una determinación óptima de la capacidad, se empleó la metodología que considera relaciones con factores como mercado, materia prima, tecnología e inversión, los cuales sirvieron de apoyo para identificar el límite superior e inferior de la capacidad, evidenciando que ningún factor se consideró limitante para el tamaño de planta identificado.

Posteriormente tras el levantamiento de información para el análisis de diferentes fuentes bibliográficas (patentes, revistas, artículos, tesis, entre otros) y la contratación de un consultor experto en el área, se ha identificado un proceso preliminar que servirá de guía inicial para el diseño de la planta de reaprovechamiento de residuos sólidos (viruta de cuero).

Dicho proceso preliminar se ha considerado dividirlo en las siguientes unidades: Primera unidad Acondicionamiento de materia prima, esta etapa involucra desde el acopiamiento de la materia prima (virutas de cuero) hasta el refinado de la misma. Inicia con la recepción de los residuos sólidos (virutas de cuero), las cuales han sido recolectadas en la etapa de rebajado y se encuentran en el almacén de virutas de cuero, posteriormente se procede el control de parámetros humedad y pesado de las virutas de cuero en bolsas, siendo la cantidad utilizada según necesidades de la línea de producción; luego se trasladan al molino de cuchillas, donde se les agrega agua en proporción aproximada de 1:15 según características de las virutas y son trituradas hasta obtener un diámetro de $5-7 \mathrm{~mm}$, obteniendo una masa de fibrillas, que inmediatamente son almacenadas en un tanque de homogenización. La masa de fibrillas es trasladada del tanque de homogenización al refinador, cuya función es igualar el tamaño de las fibras de virutas de cuero.

Segunda unidad - Mezclado y moldeado, comprende desde la preparación de la formula química (resinas y aglomerados) hasta el moldeado de planchas. Esta etapa inicia con la preparación de la fórmula química cuyas características están en función del producto que será elaborado a partir de las planchas de cuero; la fórmula es añadida al tanque mezclador (con sistema de agitación) junto a la masa de fibrillas procedente del refinador para formar una masa homogénea, posteriormente la forma de transporte será por medio de gravedad hacia el área de moldeado donde se realizará la formación de las planchas en cajas al vacío que eliminan el agua presente. 
Tercera unidad - Prensado y secado, comprende desde el prensado hasta el secado de las planchas. Esta etapa inicia con el prensado de las planchas provenientes de las cajas al vacío realizado en la prensa hidráulica, posterior a ese proceso se trasladan las planchas hacia la máquina de rebajado para obtener el grosor deseado definido por el comprador. Una vez finalizado el rebajado, las planchas son llevadas al área de secado.

Cuarta unidad - Acabado y confección, esta etapa comprende desde el lijado de las planchas que salen del área de secado hasta el almacenamiento del producto terminado. Las planchas una vez secas son trasladadas hacia la lijadora que les otorga suavidad y un espesor más preciso, seguidamente son trasladadas al área de corte donde las dimensiones de las planchas son ajustadas a dimensiones estándares, después son desplazadas hacia al área de pintado y estampado. Por último, las planchas de cuero regenerado son transportadas hacia el almacén de productos terminados.

Seguidamente, se procedió al diseño de la distribución de la línea/planta productiva para lo cual se aplicó el análisis de factores (material, maquinaria, servicios de abastecimiento, edificio y factor cambio) que permiten recopilar información sobre los distintos aspectos que influyen en la distribución de planta.

Adicionalmente para el análisis de distribución de planta se empleó la técnica de Tabla y Diagrama Relacional de Actividades, las cuales permiten obtener una óptima distribución del área de trabajo. La técnica de la Tabla Relacional de Actividades, indica la importancia de cercanía o proximidad entre cada actividad o función para lo cual se desarrollará previamente la Tabla de Valor de Proximidad y la Tabla de Lista de Motivos para mejor entendimiento del diagrama. Se consideraron 6 motivos: 1) Secuencia de flujo de trabajo, 2) Por abastecimiento de materiales, 3) No se desea la contaminación del producto final, 4) Por ser emplear alguna maquinaria compartida, 5) Por seguridad y 6) No es necesario. Además, la escala de valores de proximidad que se ha considerado está representada de la siguiente forma: "A" significa absolutamente necesario; "I", Importante; "U", Sin importancia; por último "X", No recomendable.

A continuación de describen las consideraciones asumidas para la ubicación en cada valor de proximidad:

- Con "A" "Absolutamente Necesario" se consideran aquellas actividades que necesariamente deben estar ubicada una a lado de la otra.

- "I" "Importante" a aquellas actividades no consecutivas, pero que se encuentran en la misma unidad del proceso, tienen una separación de continuidad de un espacio entre ambas, es decir, pueden precederles una actividad que no es parte de la secuencia de esa unidad sin que intervenga con su operación.

- "U" "Sin Importancia" para las actividades que no guardan relación con la secuencia del proceso productivo, no es imprescindible su cercanía.

- "X" "No recomendable" a aquellas actividades que no deben estar ubicadas cercanas a la línea productiva debido que puedan ocasionar algún peligro para la producción a causa de acopiamiento de materiales peligrosos, preparación de mezclas químicas, etc.

Por tal motivo la Tabla relacional obtenida para la línea productiva de aprovechamiento de residuos sólidos (viruta de cuero), así como sus valores de proximidad, indican que para realizar la distribución tentativa de planta hay que tener en cuenta como actividades cercanas principalmente aquellas que tuvieron una relación de "Absolutamente necesario" e "Importante" como son por ejemplo Molido de Virutas de cuero y Homogenizado de virutas de cuero. 


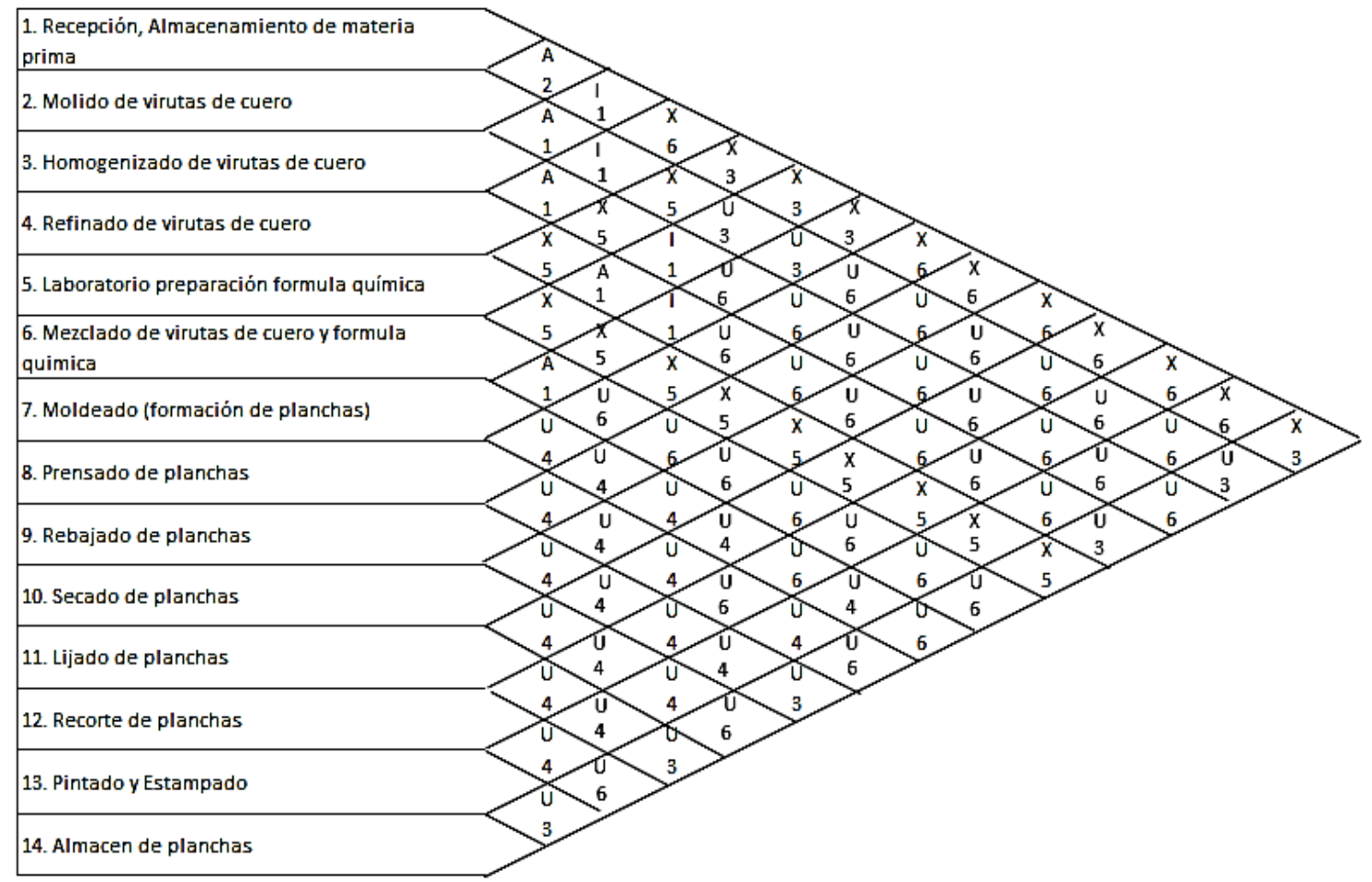

Figura 1. Tabla relacional de actividades. Fuente: (Medina Ninacondor et al., 2018)

La tabla 1 presenta el resumen de la Tabla Relacional el cual permitirá elaborar el Diagrama Relacional de Actividades (Figura 2) que representa el primer bosquejo de la distribución de planta. Para ello se ha considerado los siguiente: el valor "A" (absolutamente necesario) se representa con línea roja; valor "I" (importante) está de color verde; valor "U" está de color azul y el valor "X" (no recomendable) está de color plomo con rayas discontinuas.

Tabla 1. Valores de Proximidad para implementación de la línea productiva de cuero regenerado.

\begin{tabular}{cl}
\hline Proximidad & \multicolumn{1}{c}{ Intervalo de Proximidad } \\
\hline $\mathbf{A}$ & $(1,2) ;(2,3) ;(3,4) ;(4,6) ;(6,7)$ \\
$\mathbf{I}$ & $(1,3) ;(2,4) ;(3,6) ;(4,7)$ \\
& $(2,6) ;(2,7) ;(2,8) ;(2,9) ;(2,10) ;(2,11) ;(2,12) ;(2,13) ;(2,14) ;(3,7) ;(3,8) ;$ \\
& $(3,9) ;(3,10) ;(3,11) ;(3,12) ;(3,13) ;(3,14) ;(4,8) ;(4,9) ;(4,10) ;(4,11) ;(4,12) ;$ \\
& $(4,13) ;(4,14) ;(6,8) ;(6,9) ;(6,10) ;(6,11) ;(6,12) ;(6,13) ;(6,14) ;(7,8) ;(7,9) ;$ \\
& $(7,10) ;(7,11) ;(7,12) ;(7,13) ;(7,14) ;(8,9) ;(8,10) ;(8,11) ;(8,12) ;(8,13) ;$ \\
& $(8,14) ;(9,10) ;(9,11) ;(9,12) ;(9,13) ;(9,14) ;(10,11) ;(10,12) ;(10,13) ;$ \\
& $(10,14) ;(11,12) ;(11,13) ;(11,14) ;(12,13) ;(12,14) ;(13,14)$ \\
& $(1,4) ;(1,5) ;(1,6) ;(1,7) ;(1,8) ;(1,9) ;(1,10) ;(1,11) ;(1,12) ;(1,13) ;(1,14) ;$ \\
& $(2,5) ;(3,5) ;(4,5) ;(5,6) ;(5,7) ;(5,8) ;(5,9) ;(5,10) ;(5,11) ;(5,12) ;(5,13) ;$ \\
& $(5,14)$
\end{tabular}

Fuente: (Medina Ninacondor et al., 2018)

A partir del diagrama relacional de actividades, se realizará la distribución tentativa de planta, que cumple con los criterios establecidos en el análisis relacional. Se ha evidenciado que los equipos que conforman la 
unidad 1 y 2 (el molino, el tanque homogenizador, el refinador, el mezclador y las cajas de moldeado) deben estar ubicados de forma continua.

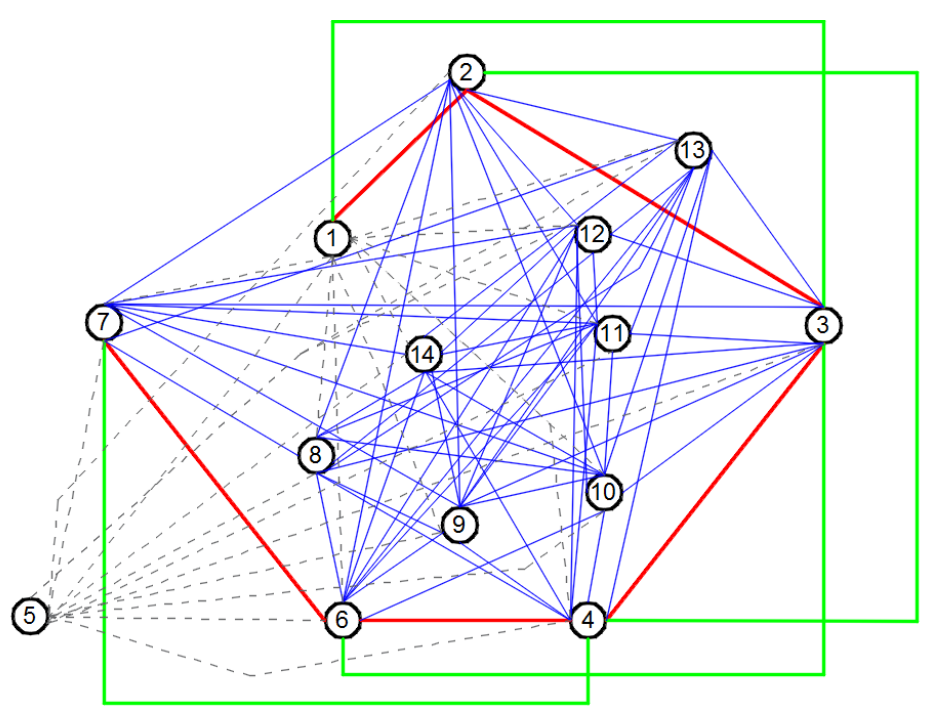

Figura 2. Diagrama Relacional de Hilos Fuente: (Medina Ninacondor et al., 2018)

Seguidamente fue necesario empelar el balance de línea poder determinar el personal mínimo requerido según las estaciones que se diseñaran para la línea productiva del proyecto. Inicialmente se procedió a determinar las actividades y operaciones del proceso y, la relación respecto a la precedencia entre ellos; seguidamente se determinó el tiempo aproximado de desarrollo de cada uno (Tabla 2) para lo cual se ha considerado la ficha técnica de los equipos y las distancias existentes en la zona, lo cual proporciono un tiempo tentativo que posteriormente tendrá que ser validado en el proceso. Cabe resaltar que no se ha considerado los tiempos de descanso y secado ya que, a pesar de ser las operaciones que demandan más tiempo en el proceso, ambos son procesos donde no se experimentan cambios (transformaciones de estado) en las planchas de cuero regenerado por lo cual en éstas áreas sólo se realiza el secado a temperatura ambiente. Los días de trabajo mensuales en un mes equivalen a 24 días laborables con jornadas de $8 \mathrm{~h}$ de lunes a viernes y $4 \mathrm{~h}$ los días sábados donde se producirá aproximadamente 116 lotes de 7 planchas cada uno.

Tabla 2. Actividades y operaciones

\begin{tabular}{lccc}
\hline \multicolumn{1}{c}{ Descripción } & Actividades & Precedencia & Tiempo (min) \\
\hline Proceso de molido & $\mathrm{A}$ & - & 33 \\
Proceso de homogenización & $\mathrm{B}$ & $\mathrm{A}$ & 11 \\
Proceso de refinado & $\mathrm{C}$ & $\mathrm{B}$ & 10 \\
Proceso de mezclado & $\mathrm{D}$ & $\mathrm{C}$ & 72 \\
Formación de planchas & $\mathrm{E}$ & $\mathrm{D}$ & 24 \\
Proceso de prensado & $\mathrm{F}$ & $\mathrm{E}$ & 15 \\
Descanso & $\mathrm{G}$ & $\mathrm{F}$ & 1622 \\
Proceso de rebajado & $\mathrm{H}$ & $\mathrm{G}$ & 20 \\
Secado & $\mathrm{I}$ & $\mathrm{H}$ & 540 \\
Proceso de lijado & $\mathrm{J}$ & $\mathrm{I}$ & 18 \\
Proceso de recorte & $\mathrm{K}$ & $\mathrm{J}$ & 11 \\
Proceso de pintado & $\mathrm{L}$ & $\mathrm{K}$ & 11 \\
\hline Total & & & 225 \\
Fuente: (Medina Ninacondor et al., 2018) & & &
\end{tabular}


A continuación, se realizó el cálculo de los indicadores requeridos para el balance de línea.

Productividad de la planta (unidades al día)

$$
\frac{\text { Lotes a producir al mes }}{\text { Numero de días de producción }}=\frac{116 \text { lotes }}{24 \text { días }}=4.8333 \text { lote } / \text { día }
$$

Tasa de producción deseada (unidades por minuto)

$$
\frac{\text { Productividad de la planta }}{8 h \times 60 \mathrm{~min}}=\frac{4.8333}{8 \times 60}=0.010069 \mathrm{u} / \mathrm{min}
$$

Tiempo de ciclo (minutos por unidad)

$$
\frac{1}{\text { Tasa de producción deseada }}=\frac{1}{0.010069}=99.31 \mathrm{~min} / \mathrm{lote}
$$

Mínimo teórico de Estaciones

$$
\frac{\text { Tiempo total }}{\text { Tiempo de ciclo }}=\frac{225}{99.31}=2.3 \approx 3 \text { estaciones como mínimo }
$$

Eficiencia (\%)

$$
\frac{\text { Tiempo total } \times 100}{\text { Tiempo de ciclo } \times \text { mín.teórico de estac. }}=\frac{225 \times 100}{99.31 \times 3}=75.5 \%
$$

Tiempo ocioso

$$
(\text { Tiempo de ciclo } \times \text { mínimo teórico de Estaciones })-\text { tiempo total }=(99.31 \times 3)-225=73.93 \mathrm{~min}
$$

Retraso (\%)

$$
100-\text { Eficiencia }(\%)=24.5 \%
$$

A través de este análisis se ha evidenciado que la línea productiva tiene dos operaciones (descanso y secado) que va a demandar más tiempo por lo cual va a retrasar el proceso y que no han sido considerados por representar esperas, por otro lado, a través del balance realizado se observa que existe un $24.5 \%$ de capacidad instalada sin utilizar lo cual tiene que ser optimizado al momento de ser implementado.

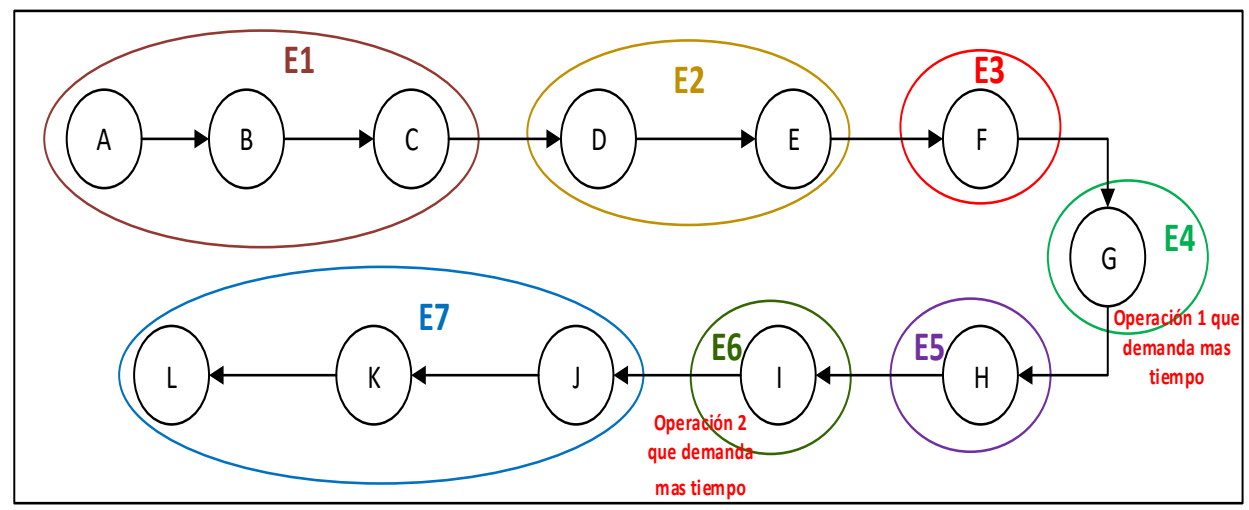

Figura 3. Diagrama de distribución de operarios según agrupación de actividad Fuente: (Medina Ninacondor et al., 2018) 
Se ha asignado 5 operarios, 2 de ellos para la estación E1, E2 y E3 por ser las tres primeras estaciones continuas y los otros 3 alternarán actividades en las demás estaciones.

Otra herramienta que se ha aplicado para determinar el espacio requerido para la instalación de la planta fue el Método de Guerchet, para lo cual previamente se identificó el número total de maquinaria y equipo denominados elementos estáticos y el número de operarios u equipo de acarreo denominados elementos móviles (Tabla 3).

Tabla 3. Dimensiones de elementos estáticos y móviles requeridos por la línea/planta productiva

\begin{tabular}{|c|c|c|c|c|c|}
\hline Elementos Estáticos & $\begin{array}{c}\mathrm{n} \\
\left(\mathrm{N}^{\circ} \text { de elementos Móviles }\right. \\
\text { o estáticos de un tipo })\end{array}$ & $\begin{array}{l}\mathrm{N}\left(\mathrm{N}^{\circ} \text { de }\right. \\
\text { lados })\end{array}$ & $\begin{array}{l}\mathrm{L}(\mathrm{m}) \\
\text { (Largo) }\end{array}$ & $\begin{array}{c}\text { A (m) } \\
\text { (Ancho) }\end{array}$ & $\begin{array}{l}\mathrm{H}(\mathrm{m}) \\
\text { (Alto) }\end{array}$ \\
\hline Molino de cuchillos & 1 & 1 & 1.20 & 1.20 & 1.70 \\
\hline $\begin{array}{l}\text { Tanque de } \\
\text { Homogenización }\end{array}$ & 1 & 1 & 1.40 & 1.40 & 1.40 \\
\hline Refinador de pulpa & 1 & 1 & 2.15 & 0.95 & 1.50 \\
\hline $\begin{array}{l}\text { Bomba centrífuga con } \\
\text { acoplamiento helicoidal }\end{array}$ & 1 & 1 & 1.00 & 1.00 & 0.50 \\
\hline Mezclador & 1 & 1 & 1.40 & 1.40 & 1.40 \\
\hline $\begin{array}{l}\text { Moldeador (cajas de } \\
\text { desagüe) }\end{array}$ & 1 & 2 & 2.50 & 3.20 & 1.00 \\
\hline $\begin{array}{l}\text { Bomba de vacío } \\
\text { Elementos Móviles }\end{array}$ & 2 & 1 & 1.00 & 1.00 & 0.50 \\
\hline Operarios & 5 & 2 & & & 1.65 \\
\hline
\end{tabular}

Este cálculo proporciona el área total requerido en base a la suma de las siguientes superficies parciales: Superficie Estática (Ss): Comprende el área ocupada por la máquina. Superficie de Gravitación (Sg): Se refiere a la superficie empleada por el operario y el material acopiado. Superficie de Evolución (Se): Involucra el área necesaria para circulación y desplazamientos del personal (Nievel, 2004).

Para el cálculo de las superficies parciales será necesario el coeficiente de evolución (K) que será obtenido a continuación:

$$
\begin{gathered}
k=\frac{\text { Altura media de elementos móviles }}{\text { Altura media de elementos estáticos }} \\
k=\frac{1.65}{2 \times 1.29}=0.64
\end{gathered}
$$

Como siguiente paso será necesario calcular la superficie total, por lo que se procede a la sumatoria de las superficies parciales. Los cálculos desarrollados se muestran a continuación (Tabla 4), de esta forma, se obtiene que se requiere un área aproximada de $85.77 \mathrm{~m} 2$.

Finalmente, para la elaboración de los planos correspondientes de la línea/planta productiva se ha requerido un estudio general de las instalaciones eléctricas actuales de la planta para corroborar si alguno de los tableros de distribución actual podría albergar la nueva demanda o si se requerirá la instalación de un nuevo tablero. El desarrollo de este estudio ha tenido como base la Ley de Concesiones Eléctricas Decreto Ley $\mathrm{N}^{\circ} 25844$, asimismo el Código Nacional de Electricidad, además de la Norma $\mathrm{N}^{\circ}$ DGE 017 AI-1/1982 de Alumbrado de Interiores y Campos Deportivos. Para la determinación de la demanda de la línea productiva para aprovechamiento de residuos sólidos se ha requerido datos técnicos de corriente y potencia en cada estación de trabajo, además de requerimientos de seguridad eléctrica como interruptores termo magnéticos y diferenciales, puesta a tierra, entre otros. 
Posterior al análisis anterior se determina que considerando un factor de simultaneidad de encendido de las maquinas en un $70 \%$, la demanda proyectada para la implementación de la línea/planta productiva será de $104.106 \mathrm{~kW} / \mathrm{h}$ (Tabla 5) requiriendo un nuevo tablero de distribución ya que los actuales de la planta se encuentran en su límite.

Tabla 4. Análisis y cálculo de Área Total

\begin{tabular}{lcccc}
\hline \multicolumn{1}{c}{ Elementos Estáticos } & $\begin{array}{c}\mathrm{Ss}(\mathrm{m} 2) \\
\mathrm{L} \mathrm{x} \mathrm{A}\end{array}$ & $\begin{array}{c}\mathrm{Sg}(\mathrm{m} 2) \\
\mathrm{Ss} \mathrm{x} \mathrm{N}\end{array}$ & $\begin{array}{c}\mathrm{Se}(\mathrm{m} 2) \\
(\mathrm{Ss}+\mathrm{Sg}) \mathrm{K}\end{array}$ & $\begin{array}{c}\mathrm{St}(\mathrm{m} 2) \\
(\mathrm{Ss}+\mathrm{Sg}+\mathrm{Se}) \mathrm{n}\end{array}$ \\
\hline $\begin{array}{l}\text { Molino de cuchillos } \\
\begin{array}{l}\text { Tanque de } \\
\text { homogenización }\end{array}\end{array}$ & 1.44 & 1.44 & 1.84 & 4.72 \\
$\begin{array}{l}\text { Refinador de pulpa } \\
\begin{array}{l}\text { Bomba centrífuga con } \\
\text { acoplamiento }\end{array}\end{array}$ & 1.96 & 1.96 & 2.51 & 6.43 \\
helicoidal & 1.04 & 2.04 & 2.61 & 6.69 \\
$\begin{array}{l}\text { Mezclador } \\
\begin{array}{l}\text { Moldeador (cajas de } \\
\text { desagüe) }\end{array}\end{array}$ & 1.96 & 1.00 & 1.28 & 3.28 \\
$\begin{array}{l}\text { Bomba de vacío } \\
\quad \text { Elementos Móviles }\end{array}$ & 1.00 & 1.00 & 2.51 & 6.43 \\
$\begin{array}{l}\text { Operarios } \\
\text { Área total }\end{array}$ & 0.5 & 1.00 & 15.36 & 39.36 \\
Fuente: (Medina Ninacondor et al., 2018) & & & 1.28 & 6.56 \\
\end{tabular}

Tabla 5. Cálculo de la demanda eléctrica proyectada para la línea productiva

\begin{tabular}{|c|c|c|c|c|c|}
\hline Descripción & $\begin{array}{l}\text { Corriente de } \\
\text { operación (A) }\end{array}$ & $\begin{array}{c}\text { Carga Unitaria } \\
(\mathrm{kW})\end{array}$ & Cant. & $\begin{array}{c}\text { Factor de } \\
\text { simultaneidad }\end{array}$ & $\begin{array}{c}\text { Máxima } \\
\text { Demanda }(\mathrm{kW})\end{array}$ \\
\hline Molino picador & 6.3 & 3.73 & 1 & 0.7 & 2.61 \\
\hline Refinador de doble disco & 62.46 & 37 & 1 & 0.7 & 25.9 \\
\hline Bomba de vacío 7.5HP & 9.45 & 5.6 & 1 & 0.7 & 3.92 \\
\hline $\begin{array}{l}\text { Bomba centrífuga con } \\
\text { acoplamiento helicoidal }\end{array}$ & 2.52 & 1.49 & 1 & 0.7 & 1.04 \\
\hline $\begin{array}{l}\text { Bomba centrífuga con } \\
\text { acoplamiento helicoidal }\end{array}$ & 2.52 & 1.49 & 1 & 0.7 & 1.04 \\
\hline Mezclador & 1.58 & 0.94 & 1 & 0.7 & 0.66 \\
\hline Prensa & 18,76 & 11.11 & 1 & 0.7 & 7.777 \\
\hline Rebajadora & 58.75 & 34.8 & 1 & 0.7 & 24.36 \\
\hline Cadena Aérea & 16.26 & 9.63 & 1 & 0.7 & 6.741 \\
\hline Lijadora & 32.5 & 19.25 & 1 & 0.7 & 13.475 \\
\hline Pigmentadora & 39.99 & 23.69 & 1 & 0.7 & 16.583 \\
\hline \multicolumn{5}{|c|}{ Total máxima demanda del tablero $(\mathrm{kW})$} & 104.106 \\
\hline
\end{tabular}




\section{RESULTADOS Y DISCUSIÓN}

A partir del análisis desarrollado para el diseño de una línea/planta productiva para el reaprovechamiento de residuos sólidos (viruta de cuero, se alcanzaron los siguientes resultados:

La ubicación óptima que se ha seleccionado cuenta con un área disponible de $87.11 \mathrm{~m}^{2}$ adecuada para la implementación de la línea, no requiere alta inversión por abastecimiento de servicios (eléctrico y agua) ya que incurrirá sólo en gastos por conectividad y mano de obra, presenta un recorrido total del proceso de $216 \mathrm{~m}$ y los costos administrativos que se requerirán para la implementación serán equivalentes a S/. 2000.00, destinados para el estudio de suelos y anclaje de la maquinaria.

Es importante tener claras las consideraciones sobre las cuales se definirá la capacidad instalada de la línea/planta productiva ya que en base a esa capacidad se realizará el dimensionamiento. La capacidad instalada para el proyecto se ha tomado en base a un crecimiento futuro del $37 \%$ de la producción de la empresa, lo cual generaría $6600 \mathrm{~kg}$ de residuos sólidos (viruta de cuero) para aprovechar.

Se identificó previamente el proceso preliminar definiendo cuatro unidades: Acondicionamiento de materia prima; mezclado y moldeado; prensado y

Secado y; acabado y confección.

Para la realización del estudio se ha empleado herramientas de Tabla Relacional y Diagrama Relacional de Hilos, balance de línea y el método de Guerchet mediante los cuales se pudo obtener la distribución final que tendrá la línea/planta productiva para el reaprovechamiento de residuos sólidos (viruta de cuero) (Figura 4), considerando previamente áreas, ambientes, vías de acceso, distancias establecidas por norma, servicios, secuencia e interacción entre actividades y la maquinaria necesaria para la obtención del producto final (cuero regenerado).

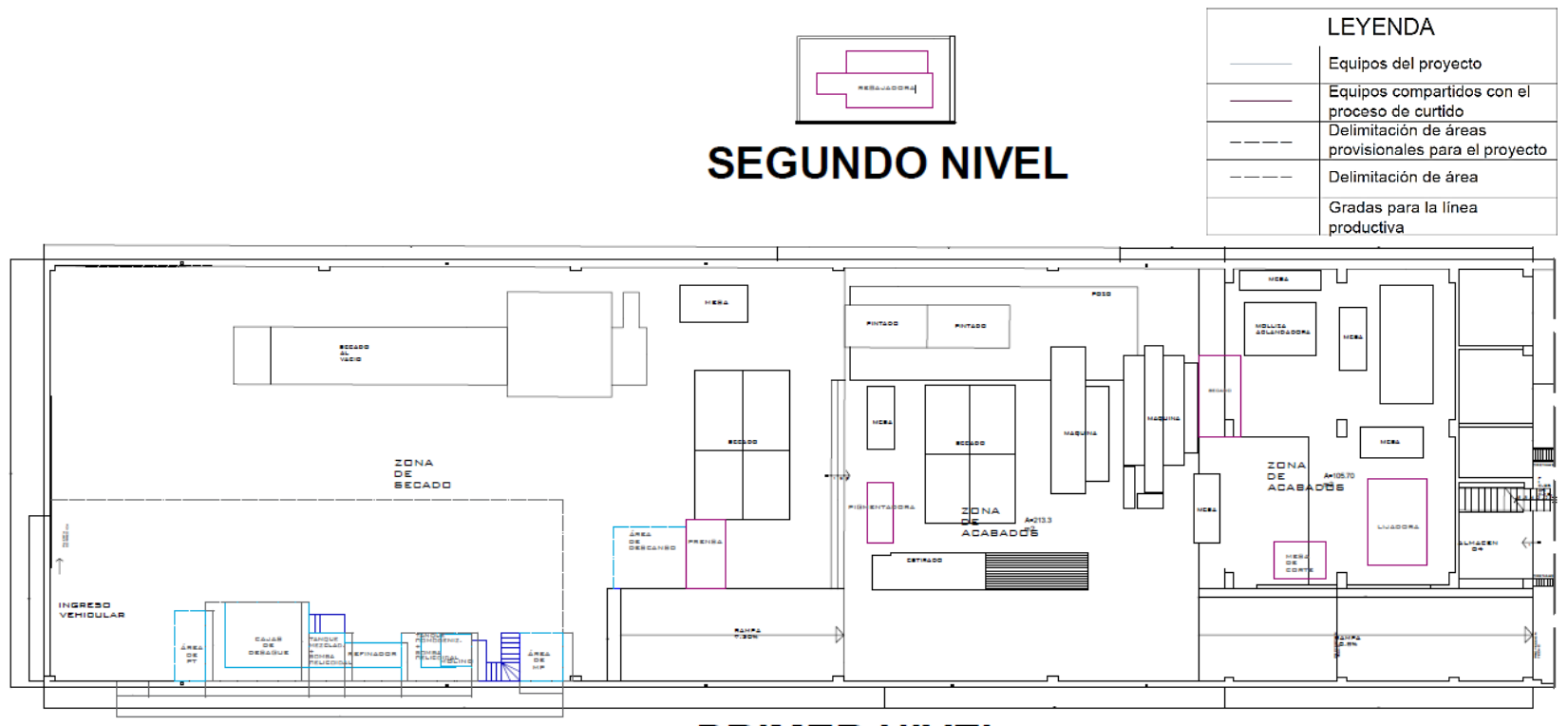

PRIMER NIVEL

Figura 4. Layout preliminar de la línea productiva para el aprovechamiento de residuos sólidos (viruta de cuero) Fuente: (Medina Ninacondor et al., 2018)

Para la elaboración de los planos eléctricos previamente se analizó el Código Nacional de Electricidad; así como del Reglamento Nacional de Edificaciones, además se ha considerado la demanda total proyectada para la implementación de la línea que anteriormente ha sido calculada y equivale a $104.106 \mathrm{~kW} / \mathrm{h}$. Se ha 
requerido la instalación de un nuevo tablero que pueda albergar esta nueva demanda, en la Figura 5 se observa las conexiones eléctricas para maquinaria, luminarias, tomacorrientes e interruptores.

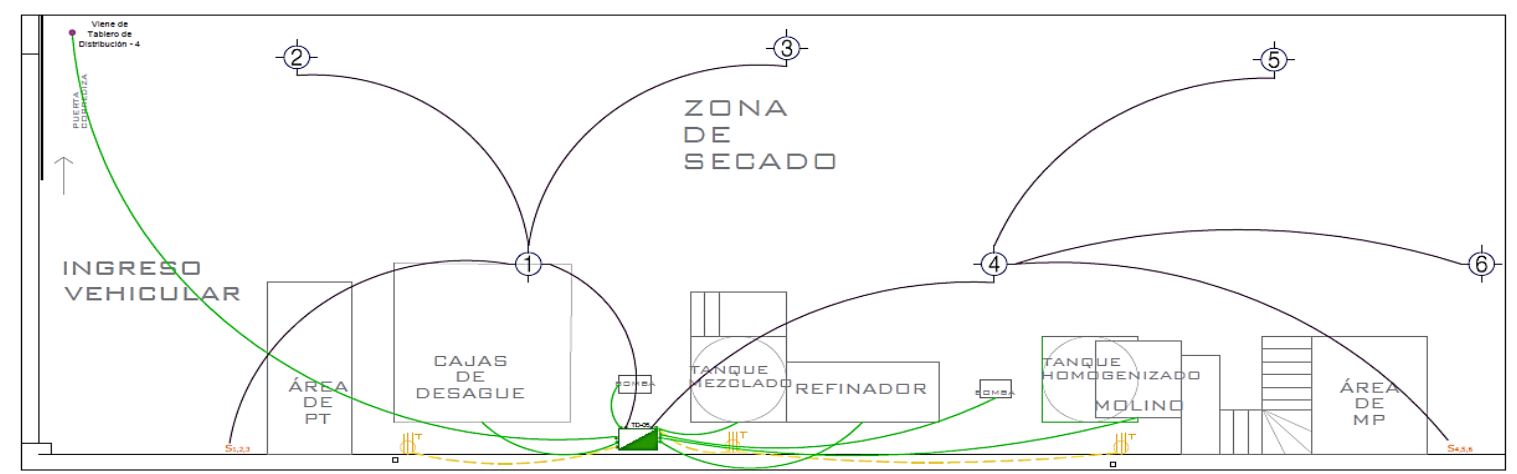

\begin{tabular}{|c|c|}
\hline SIMBOLO & DFSCRITGION \\
\hline oSa,b,co & Interruptor uripolar simple 6 triple \\
\hline$-\frac{1}{-}-$ & Luminaria con lampara ahorradora do $20 \mathrm{~W}$ (techo) \\
\hline$\Delta$ & Tablero de distribución de tuerza (TD) \\
\hline$\stackrel{5}{7}$ & Pozo de Puesta a Ticrra \\
\hline 㨁 & Iomacorriente dohle de $380 \mathrm{~V}$ tritásico \\
\hline$\ldots$ & 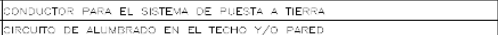 \\
\hline & IRCLITO OE TOMACDREIENTE EN LA PAFEO Y/O FISO \\
\hline
\end{tabular}

Figura 5. Diseño de las instalaciones eléctricas Fuente: (Medina Ninacondor et al., 2018)

Se ha empleado un software a través del cual se ha realizado el modelado de la línea/planta industrial para el reaprovechamiento de residuos sólidos (viruta de cuero). Autocad Plant 3D es un programa de diseño asistido por computadora que permite realizar dibujos en tres dimensiones, este software ha hecho posible crear en su totalidad el diseño de la línea productiva asignando dimensiones reales y permitiendo visualizar el proyecto desde diferentes perspectivas.

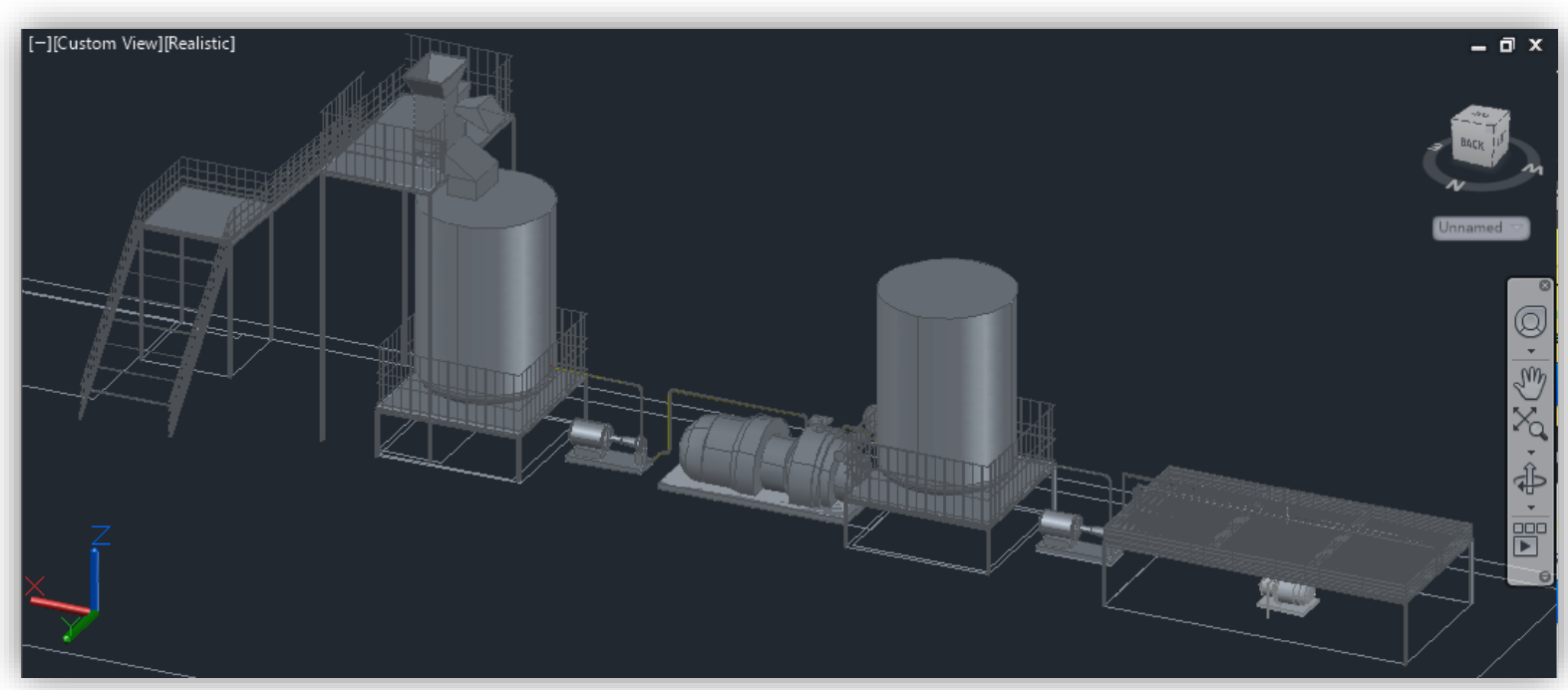

Figura 6. Diseño de la Planta modelada en 3D

Fuente: (Medina Ninacondor et al., 2018) 


\section{CONCLUSIONES}

Se ha corroborado y demostrado que para un adecuado diseño de planta es necesario la aplicación de diferentes técnicas y herramientas ingenieriles para el diseño de planta dentro de ellos el análisis de factores para identificar la localización (cercanía a la materia prima, abastecimiento de servicios, recorrido del proceso, gastos administrativos, entre otros) y factores para el diseño de la distribución de planta (material. Maquinaria, servicios, edificio y cambio) (Díaz Garay \& Jarufe Zedán, 2007). El uso de herramientas como el método de Guerchet y Muther (Diagrama y Tabla Relacional), junto con el Reglamento Nacional de Edificaciones, han permitido obtener la distribución de espacios, así como la ubicación de la maquinaria necesaria para la implementación de la línea productiva (Konz, 1987) (Sapag Chain, 2011). El área necesaria para la implementación de la planta productiva deberá tener $85.77 \mathrm{~m}^{2}$ aproximadamente, para el suministro del servicio eléctrico se requerirá la instalación de un nuevo tablero con tensión de 380/220 V trifásico ya que los existentes en la planta se encuentran en su límite, el personal necesario para la nueva línea productiva rotara actividades para optimizar la mano de obra y disminuir costos. Finalmente, el diseño de la línea productiva ha sido desarrollado en base a una capacidad de 6600 $\mathrm{kg}$ de residuos sólidos (viruta de cuero), ante un crecimiento proyectado por la empresa en su producción del 37\%, representando 812 planchas de cuero regenerado mensual que se pueden obtener aproximadamente.

\section{REFERENCIAS}

Álvarez, A. M. (2009). Desarrollo de un método de distribución física aplicable en las industrias Ecuatorianas (Tesis de Pregrado). Escuela Superior Politécnica del Litoral, Guayaquil.

Díaz Garay, B., Jarufe Zedán, B., \& Noriega Araníbar, M. T. (2007). Disposición de planta. Lima: Fondo Editorial.

Contreras, M. (2004). Formulación y evaluación de proyectos (3 ed.). Bogotá: Editorial Unad.

Dirección General de Salud Ambiental. (2004). Marco institucional de los residuos sólidos en el Perú. Lima: SINCO Editores.

Konz, S. (1987). Diseño de Instalaciones Industriales. México: Limusa S.A.

Nievel, F. (2004). Ingeniería industrial, métodos, estándares y diseño del trabajo (10 ed.). México: Mc Graw-Hill/Interamericana Editores.

Sapag Chain, N. (2011). Proyectos de Inversión Formulación y Evaluación. Santiago de Chile: Graw Hill.

\section{SEMBLANZA DE LOS AUTORES}

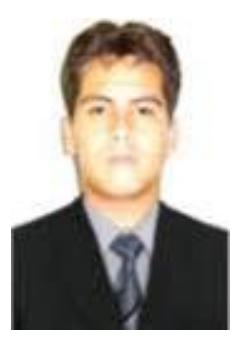

Jose A. Aguilar Franco: Obtuvo el grado de Ingeniero Industrial en la Universidad Católica San Pablo, Perú, donde actualmente es profesor titular, además de Ingeniero de Investigación del Programa de Iniciación Científica, formulador de proyectos y Coordinador General del Instituto de Energía y Medio Ambiente de la Universidad Católica San Pablo. Desarrolló sus estudios de maestría en la Pontificia Universidad Católica de Río de Janeiro (PUC - Río) y Universidad Nacional de San Agustín, Perú (UNSA). Ha desarrollado una estancia de investigación en Italia para Capacitación en Uso de Maquinarias Ecológicas. Cuenta con experiencia como asesor de Tesis, además 
trabaja como Formulador de Proyectos bajo la modalidad de concurso a nivel Nacional en proyectos de Investigación e Innovación convocadas por el Estado Peruano.

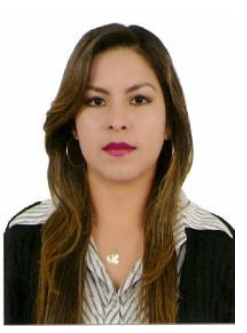

Katherine E. Medina Ninacondor: Obtuvo el grado de Ingeniera Industrial en la Universidad Católica San Pablo, Perú. Actualmente formula, gestiona y apoya en proyectos de inversión e investigación en innovación mediante convenios en asociación a través del Instituto de Energía y Medio Ambiente (IEM) de la Universidad Católica San Pablo con el Estado Peruano y diferentes empresas privadas.

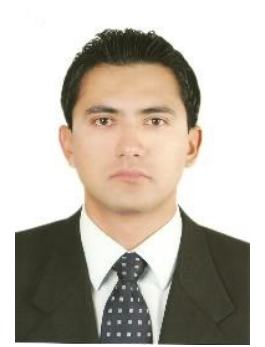

Jeanmarco Villegas Alvarez: Obtuvo el grado de Licenciado en Administración de Negocios en la Universidad Católica de San Pablo, Perú, donde actualmente es profesor titular. Desarrollo sus estudios de maestría en Administración de Empresas (MBA) en la Universidad Peruana de Ciencias Aplicadas (UPC). Cuenta con experiencia como especialista en dirección de entidades financieras por FUNCASEspaña, especialista en planeamiento de negocios y gestión estratégica y, Gestor de Proyectos de Innovación y Sociales 